\title{
Realize: Resource Management for Soft Real-Time Distributed Systems *
}

\author{
P. M. Melliar-Smith, L. E. Moser, V. Kalogeraki, P. Narasimhan \\ Department of Electrical and Computer Engineering \\ University of California, Santa Barbara, CA 93106
}

\begin{abstract}
The Realize system simplifies the development of complex applications by separating the application programming from the management of resources for soft real-time CORBA applications, and from the replication of CORBA objects to provide high availability and fault tolerance. Realize uses totally ordered multicast messages to maintain consistency of the states of the object replicas, and adjusts the degree of replication dynamically as resource availability changes. By measuring the usage of the resources and by monitoring the behavior of application objects, Realize allocates objects to processors and migrates objects between processors to balance the load on the processors. Realize employs a least-laxity scheduling strategy that meets soft real-time deadlines for tasks operating across multiple processors. Thus, with Realize, the difficult issues of distribution, real-time scheduling, load balancing, replication, consistency, and fault detection and recovery are hidden from the application programmer.
\end{abstract}

\section{Introduction}

Major defense systems are among the most complex systems ever constructed. In some cases the defense application is inherently difficult; in other cases the application is not difficult in itself but is surrounded by so many rules and special cases that the resulting system becomes very complex. Many defense applications must be networked with other defense applications, which further increases the complexity. Each defense application is difficult, and requires considerable time, to program. In a future information war, the speed with which new applications can be developed, and existing applications can be modified, can be critical to success. Moreover, the application must be programmed by experts in the application domain, and such experts are not easy to find.

${ }^{*}$ This research has been sponsored by the Defense Advanced Research Projects Agency in conjunction with the Office of Naval Research under Contract N00174-95-K-0083.
The situation is even worse. Defense systems must satisfy additional requirements of fault tolerance, realtime performance, and security. Each of these additional requirements is difficult and time consuming to program, and must be programmed by experts in those specific topics; such experts are also scarce. Unfortunately, existing methodologies intermix the mechanisms for fault tolerance and real time with the algorithms for the defense application itself. This causes the applications, which are already quite complex, to become even more complex. The application development timescales increase, because testing for fault tolerance and real time is difficult, which makes it even more difficult to find programmers who are expert both in the application domain and in fault tolerance and real time.

Clearly, what is needed is a methodology that enables application programmers, who are expert in the domain of their defense application, to program that application as cleanly as possible without having to worry about additional considerations. Similarly, the mechanisms that provide fault tolerance and real time capabilities should be programmed and tested separately from the application, once only, by experts in those domains, and then used for a variety of defense applications.

The Realize system $[7,8,11]$ achieves that separation. Using Realize, the application programmer programs a fault-tolerant, soft real-time distributed application, without having to address the difficult issues of distribution, real-time scheduling, load balancing, replication, consistency, fault detection and recovery. Realize operates on standard commercial off-the-shelf (COTS) operating systems and with standard unmodified COTS implementations of CORBA [19], preserving the portability, location transparency and interoperability that CORBA provides but augmenting those features with fault tolerance and soft real-time performance.

Thus, the Realize system provides:

- Support for simple programming of soft real-time fault-tolerant distributed applications 


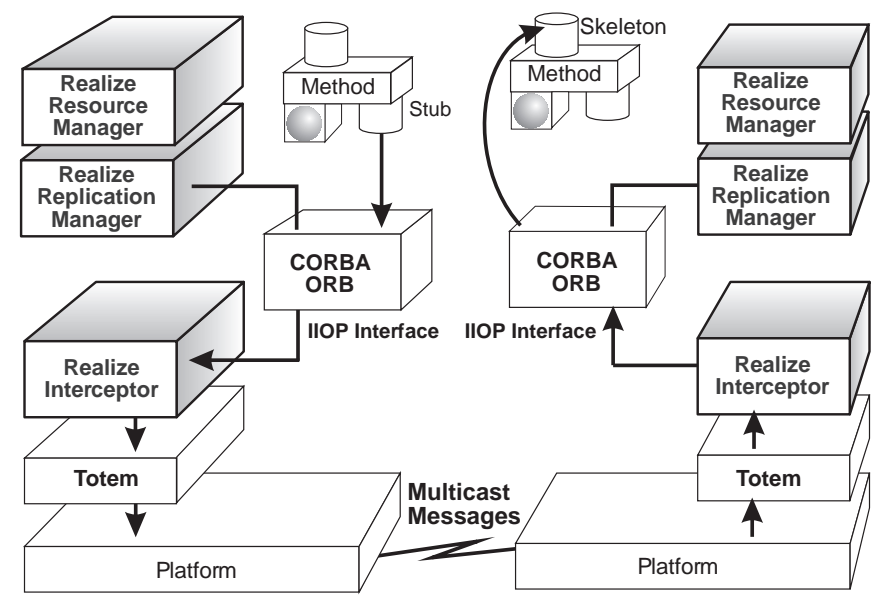

Figure 1: The structure of the Realize system, which includes the Replication Manager, the Resource Manager and the Interceptor.

- Resource management to balance the load on the resources, to schedule the tasks, and to meet soft real-time objectives

- Replication of objects and maintenance of strong replica consistency for high availability and fault tolerance.

The structure of the Realize system is shown in Figure 1. The Object Request Broker (ORB) of CORBA packages the method invocations and responses into messages, formatted according to the Internet Inter-ORB Protocol (IIOP), and subsequently passes those messages to TCP/IP for communication to other CORBA objects over the same ORB or over different ORBs. The Interceptor [14], however, intercepts the IIOP messages before they reach TCP/IP, and diverts them instead to the Replication Manager, which multicasts them to the replicas of the objects using the Totem group communication system [12].

The Resource Manager controls the allocation of resources within the distributed system. It monitors the behavior of the application objects during operation, measuring the utilization of the resources and the durations of the method invocations, and uses this information to balance the loads on the resources by moving objects, and to drive a least-laxity soft real-time scheduler. The Resource Manager is itself implemented as a collection of CORBA objects and, thus, benefits from CORBA's interoperability and Realize's fault tolerance.

The Replication Manager transparently replicates the objects of the application program to provide high availability and fault tolerance with minimal modification of those objects. Both client and server objects can be replicated, and objects can act as both clients and servers. The Replication Manager translates invocations of methods on an object group into invocations of methods on the individ- ual object replicas, and maintains the consistency of those replicas, even in the presence of faults.

\section{Resource Management in Realize}

The structure of the Realize Resource Management System is shown in Figure 2. A single Resource Manager, replicated for fault tolerance, works in concert with Profilers and Schedulers, located on each processor. The Profilers monitor the behavior of the application objects and measure the current load on the processors' resources. The Resource Manager maintains a repository of data obtained from the Profilers, replicates and distributes the objects across the processors, and moves objects when necessary to maximize the system utility and to maintain a uniform load on the resources. The Schedulers exploit information collected by the Resource Manager to schedule tasks to meet soft real-time deadlines, using a least-laxity scheduling algorithm. The actual replication of objects is provided by the Replication Manager.

The Resource Manager is responsible for assigning objects to processors and for moving objects from one processor to another. As new applications are introduced into the system, the Resource Manager determines, using the Profilers' information, whether it can satisfy their requests and then allocates resources to them accordingly. During operation, the Resource Manager might determine that a resource is overloaded or that a task is not meeting its deadlines, necessitating reallocation of the replicas or reduction of the degree of replication. The Resource Manager determines the number of replicas that are needed to satisfy reliability requirements. If a processor or other resource is lost because of a fault, the Resource Manager might need to reallocate the replicas to maintain a sufficient degree of replication to meet those requirements.

Thus, the soft real-time performance objectives of the Resource Manager are:

- To increase the probability of satisfying soft real-time response time requirements for each application task

- To balance the load of the resources by allocating the application objects to the processors and reallocating them as necessary

- To determine the degree of replication of the objects, as well as the placement of the object replicas, based on the relative importance of the application objects to the tasks.

The Resource Manager operates under the following assumptions:

- The behavior of each method invocation is independent of the context. Thus, the processing time of each method and of methods invoked by a method, and the 


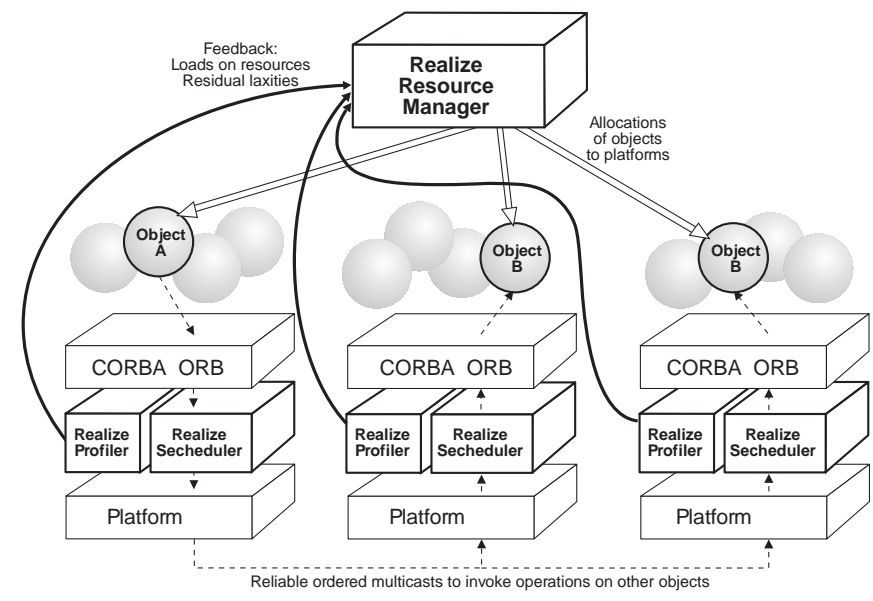

Figure 2: The structure of the Realize Resource Management System, including the Resource Manager, the Profilers and the Schedulers.

communication costs to invoke those methods, do not depend on when or why the method is invoked.

- Each resource is used for multiple activities, performed independently and asynchronously, and each such activity individually contributes only a small proportion of the total load on the resource.

- All resources are lightly loaded, with processor utilizations that are typically less than 0.5 and laxities that are typically at least half the time until the deadline. These margins suffice to accommodate statistical fluctuations in the load with acceptable probability.

The first assumption may make the Resource Manager unsuitable for systems in which nominally equivalent tasks involve substantially different numbers of operations. Well-understood deviations from this assumption can be handled by providing multiple methods to represent different behaviors. In the absence of specific information about the way in which processing times depend on task data, the use of mean processing times and an assumption of independence is the best that can be achieved.

\subsection{Feedback Loops}

The Resource Manager uses a three-level feedback loop structure:

- The first level (milliseconds), shown in Figure 3, uses measurements of elapsed time. to refine the estimated residual laxity of executing tasks, which are used by the least laxity scheduler.

- The second level (seconds), shown in Figure 3, uses measurements of elapsed time and measurements of resource loads to refine the initial estimates of the laxity for the tasks as they start.
- The third level (several seconds), shown in Figure 4, uses measured resource loads and measured residual laxities to revise the allocation of objects to processors.

\subsection{Information Base}

Upon initialization, the Resource Manager uses a configuration file to define the physical configuration available to it, including the processors and communication networks along with their specific characteristics (processing speed, memory size, network bandwidth). For each such resource, the Resource Manager periodically receives current load information (processing load, available memory, communication load) from the Profilers on the individual processors. The Resource Manager uses this information to adapt to changing system conditions.

For each application task, the Resource Manager maintains a list of the method invocations required for that task, a deadline for completion of the task, and an importance metric that is used to decide which tasks should be abandoned in the event of system overload.

For each method of each object, the Resource Manager maintains estimates of the computation time and the communication time for invocations of that method. These estimates are used to determine the initial laxity when an application task starts.

\subsubsection{Task Metrics}

An application task $t$ consists of a sequence of object invocations between an external input (or possibly a timer signal or completion of another operation) and the generation of an external result. Each task $t$ has the following metrics:

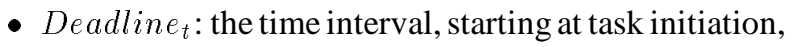
within which task $t$ should be completed, specified by the application designer

- Importance : the relative importance of task $t$, specified by the application designer, that affects the decision of which task should be abandoned in the event of system overload

- Projected_latency $y_{t}$ or $T_{t}$ : the estimated amount of time from initiation to completion of task $t$, measured by the Profiler

- Laxity $y_{t}$ the difference between Deadline $t_{t}$ and Projected_latency $y_{t}$, a measure of urgency of task $t$, calculated by the Scheduler and used by the Scheduler to schedule task $t$

- Residual_laxity : the remaining laxity when task $t$ completes, calculated by the Scheduler. 


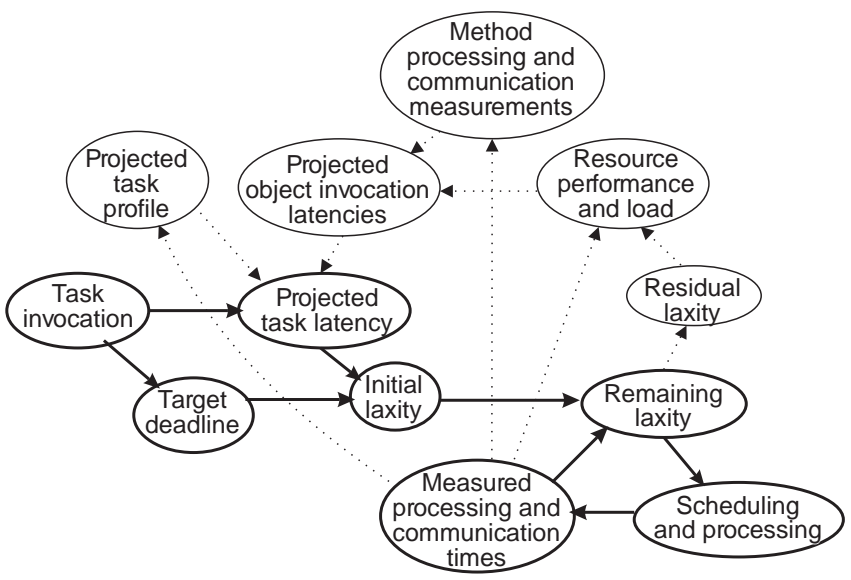

Figure 3: Least laxity scheduling. The first level feedback loop is shown with solid lines, and the second level feedback loop with dotted lines.

\subsubsection{Object and Object Replica Metrics}

For each object $i$, the Resource Manager maintains:

- Methods $s_{i}$ : the set of methods of object $i$

- Replication_type $e_{i}$ : the type of replication (active/passive) of object $i$, determined by the Resource Manager

- Replication_degree ${ }_{i}$ : the number of replicas of object $i$, determined by the Resource Manager

- Host_names $s_{i}$ : the set of hosts on which the replicas of object $i$ may be located.

\subsubsection{Method Invocation Metrics}

For each method $m$, the Resource Manager maintains:

- Object $_{m}$ : the object of which $m$ is a method

- Mean_local_time : $_{\text {: }}$ the mean time required, after receipt by a processor of a message invoking method $m$, for that processor to complete the invocation. This excludes communication time but includes queueing time and the time of embedded invocations of other methods.

- Mean_remote_time $e_{m n}$ : the mean time required for method $m$ to invoke method $n$ remotely, including communication and queueing time and also the time of embedded invocations of other methods

- Mean_communication_time $e_{m n}$ : the mean time to communicate an invocation from method $m$ to method $n$ and to communicate the response back. This is computed as the difference between Mean_remote_time $e_{m n}$ and Mean_local_time ${ }_{n}$

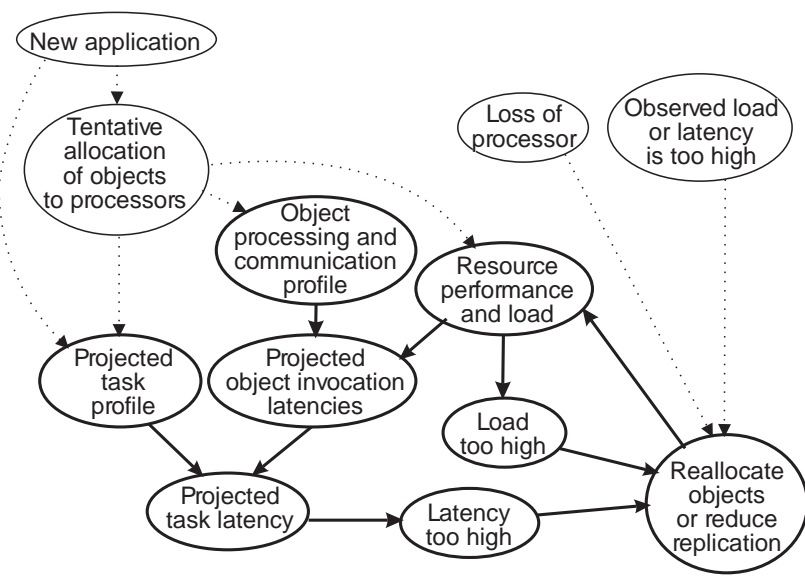

Figure 4: Object allocation and reallocation. The third level feedback loop is shown with solid lines.

- Mean_execution_time mp $_{p}$ : the mean time required for method $m$ to execute locally on processor $p$, including queueing time but excluding the time required for embedded invocations of other methods

- Mean_processing_time $e_{m p}$ or $\tau_{m p}$ : the mean time required for method $m$ to execute on processor $p$, excluding queueing time and the time required for embedded invocations of other methods

- Mean_transmission_time $e_{m c}$ or $\sigma_{m c}$ : the mean time for transmitting an invocation and a response for a method $m$ on link $c$, excluding queueing time

- Mean_invocations $s_{m n}$ : the mean number of invocations that a single invocation of method $m$ makes on method $n$.

\subsection{The Profilers}

For complex distributed applications, it is difficult to obtain accurate projections of resource requirements and other behavioral characteristics. Consequently, Realize adopts an approach based on monitoring the utilizations of the resources and the behavior of the application objects using the Profilers to supply feedback information that it uses to adjust the scheduling of tasks and the allocation of objects to processors. The Resource Manager allocates objects to processors, the objects execute and use resources, and the Profilers report resource usage to the Resource Manager, enabling it to adjust the allocations accordingly.

Each processor has its own Profiler, which is implemented between the CORBA ORB and the operating system. Each Profiler reports:

- The current utilization of resources for its processor

- The mean time required for the invocation of each remote method, determined by exponentially weighted averaging 
- The mean time and memory required for the invocation of each local method, determined by exponentially weighted averaging

- For each local method, the mean number of invocations of each other method during one invocation of that local method, determined by exponentially weighted averaging

- The mean residual laxity for each task on its completion, determined by exponentially weighted averaging.

Exponentially weighted averaging defines the expectation $E[a]$ for a measurement $a$, and a constant $c$, as follows:

$$
\begin{gathered}
E_{1}[a]=a_{1} \\
E_{i+1}[a]=E_{i}[a] *(1-c)+a_{i+1} * c
\end{gathered}
$$

where $a_{1}, a_{2}, a_{2}, \ldots$ is the sequence of measurements of $a$, the most recent measurement is weighted by $c$, and the prior expectation is weighted by $1-c$.

\subsection{The Resource Manager}

The Resource Manager estimates the Mean_local_time $m$ required to invoke method $m$ (processing, embedded invocations, queueing) by calculating the mean of the means Mean_local_time $m$ obtained from the Profilers. Similarly, it estimates the Mean_remote_time $e_{m n}$ for methods $m$ and $n$ (communication, processing, queueing, embedded invocations and overheads) and also the Mean_invocations $s_{m n}$. In addition, it calculates the Mean_communication_time mn $_{n}$ by taking the difference of the Mean_remote_time $m n$ and Mean_local_time $m$ that it calculated.

Moreover, the Resource Manager estimates the Mean execution_time $_{m p}$ for method $m$ on processor $p$, which includes queueing time but excludes embedded invocations of other methods. The estimate is derived from the Mean_local_time $m$ for method $m$, Mean_invocation $s_{m n}$ for each other method $n$ invoked by method $m$, and Mean_remote_time mn $_{\text {r }}$ for each other method $n$.

The Resource Manager then estimates the Mean processing_time $\tau_{m p}$ for each method $m$ on processor p. Let $\rho_{p}$ be the load on processor $p$. In the absence of more specific information about the application's behavior, we assume an $\mathrm{M} / \mathrm{M} / 1$ queueing model for the execution time. Thus, the Mean_processing_time $\tau_{m p}$ for method $m$ on processor $p$ is given by:

$$
\tau_{m p} \equiv\left(1-\rho_{p}\right) * \text { Mean_execution_time } e_{m p}
$$

Similarly, let $\rho_{c}$ be the load on communication link $c$. Then the Mean_transmission_time $\sigma_{m e}$ for method $m$ on communication link $c$ is given by:

$$
\sigma_{m e} \equiv\left(1-\rho_{c}\right) * M e a n_{-} \text {communication_time } m e
$$

Using a classical equilibrium flow analysis, the Resource Manager determines the mean number $x_{t m}$ of invocations of method $m$ for one execution of task $t$.

Given the Mean_processing_time $\tau_{m p}$, the Mean transmission_time $\sigma_{m e}$ for method $m$ on processor $p$ and communication link $c$, and the mean number $x_{t m}$ of invocations of method $m$ made by task $t$, the Resource Manager computes the projected latency for the entire task as:

$$
\text { Projected_latenc } y_{t} \equiv \sum_{m} \min _{p: m \in p}\left\{\frac{x_{t m} \tau_{m p}}{1-\rho_{p}}\right\}+\frac{x_{t m} \sigma_{m e}}{1-\rho_{c}}
$$

where $m \in p$ denotes that the object $i$ of which $m$ is a method executes on processor $p$. The minimum is taken over all processors $p$ that host replicas of which $m$ is a method.

The Resource Manager's estimate of the projected latency for task $t$, based on the reports of the Profilers, represents the second level of the feedback loop structure, shown by the light arrows in Figure 3. If the Resource Manager's estimate of the Projected_latenc $y_{t}$ for task $t$ is accurate, the Residual_laxit $y_{t}$ when task $t$ completes will be the same as the initial laxity for task $t$. The Resource Manager uses the ratio of the Residual_laxity $y_{t}$ to the initial laxity to adjust its estimates of the Projected_latencyt for task $t$.

\subsection{The Schedulers}

In Realize, the schedulers uses least laxity scheduling, shown as the first level of the feedback loop structure by heavy arrows in Figure 1. Least laxity scheduling is an effective strategy for scheduling tasks across multiple processors provided that the system is not overloaded. In least laxity scheduling, the laxity $L_{t}$ of a task $t$ represents a measure of urgency of the task, defined as

Laxit $_{t}=$ Deadline $_{t}-$ Remaining_projected_latency $_{t}$

where the Remaining_projected_latencyt is the estimated amount of time from the current point of task $t$ to its completion.

The effectiveness of the least laxity scheduling algorithm compared to the earliest deadline first algorithm in a distributed system can be seen by considering the following example, shown in Figure 5. Assume that tasks A and B arrive independently at processor $\mathrm{P}$. Task A consists of a single method, while task $B$ consists of methods $B_{1}$ and $B_{2}$.

The earliest deadline first algorithm is driven by the deadlines of the tasks and assigns the highest priority to the task with the earliest deadline. Consequently, task A is scheduled first on processor P and task B starts executing when task A finishes, i.e., at time $\mathrm{t}=3$ and, thus, task B misses its deadline. In contrast, the least laxity scheduling algorithm takes into consideration the 


\begin{tabular}{|c|c|c|c|c|c|c|c|c|c|c|c|}
\hline Task & Deadline & $\begin{array}{l}\text { Projected } \\
\text { Latency }\end{array}$ & \multicolumn{10}{|c|}{$\begin{array}{c}\text { Laxity } \\
\mathrm{t}=0\end{array}$} & $\mathrm{t}=1$ & $\mathrm{t}=2$ & $\mathrm{t}=3$ & $\mathrm{t}=4$ & $\mathrm{t}=5 \mathrm{t}=6 \quad \mathrm{t}=7$ & $\mathrm{t}=8$ \\
\hline $\mathrm{A}$ & 6 & 3 & 3 & 2 & 1 & 1 & 1 & & & & \\
$\mathrm{~B}_{1} \& \mathrm{~B}_{2}$ & 9 & 8 & 1 & 1 & 1 & 1 & 1 & 1 & 1 & 1 & \\
\hline
\end{tabular}
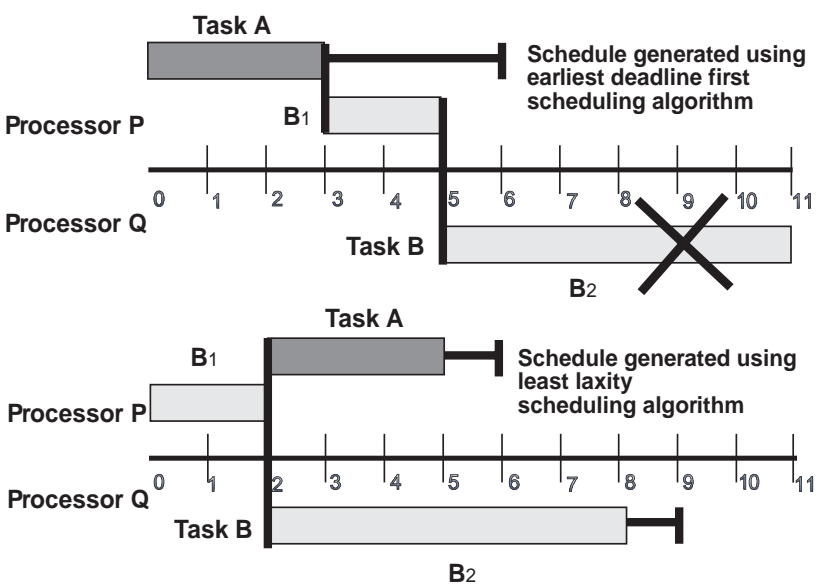

Figure 5: Least laxity scheduling finds an appropriate schedule when earliest deadline scheduling would fail.

execution time (i.e., processing and queueing time) of the tasks on the processor and schedules each method of the task independently according to the task's remaining laxity. Thus, method $\mathrm{B}_{1}$ of task $\mathrm{B}$ is scheduled first on processor $P$. When method $B_{1}$ finishes execution, both tasks have the same laxity values, task $\mathrm{A}$ is scheduled on processor $\mathrm{P}$, and method $\mathrm{B}_{2}$ of task $\mathrm{B}$ is scheduled on processor $\mathrm{Q}$. Therefore, both of the tasks meet their deadlines.

As Figure 3 shows, when a task starts, triggered by some external or internal event, the scheduler calculates a target deadline for it, and then subtracts the projected task latency, provided by the Resource Manager, to yield the initial laxity. The strategy that the Resource Manager uses to estimate the projected task latency is described below. As the application executes, the least laxity scheduler schedules the task according to the remaining laxity for that task.

The execution of the task involves the invocation of methods of objects. Each such invocation requires communication to invoke the method, processing for the method, and communication to return the results. The time required for this communication and processing is measured by the Profilers, and is reported to the Resource Manager where it is used to estimate the projected task latency. The actual time to perform the operation may be variable, and increases with the load on the processor and on the communication medium.

When a method is invoked, the time of the invocation is recorded using the operating system's high resolution timer. When the response is received, the time is again noted, and the difference between these two times is the actual time required for the method. This actual time is subtracted from the projected time, and the difference is added to the remaining laxity of the task.

The adjustment of the remaining laxity provides a feedback loop. If a task is delayed, its laxity will diminish and its scheduling priority will increase. All of the computations required to calculate the deadlines and laxities are local to the individual processors. Consequently, overheads are low and priority adjustments can be made on a microsecond-bymicrosecond basis. In contrast, the feedback loops used to estimate the projected task latency, from which the initial task laxity is derived, operate more slowly on a timescale of seconds.

The remaining laxity of the task is included in the message that communicates a remote method invocation. This allows the method to be scheduled appropriately on the remote processor hosting the object. There is no need to include a remaining laxity in a response message. As described above, the remaining laxity is adjusted using the measured time at which the response is received. When the task completes, the remaining laxity is recorded as a residual laxity, which is reported back to the Resource Manager as a measure of the current timing margins in the system.

\subsection{Allocation of Objects to Processors}

The Resource Manager maintains a global view of the application objects in the system and attempts to maintain a uniform load on all of the processors. It tries to meet the requirements of all tasks in the system, but this cannot always be achieved. Moreover, the addition of a new task can result in degradation of the performance of existing tasks. To satisfy the requirements of a new task, the Resource Manager can migrate objects to different processors or reduce the degree of replication of objects [7]. Migration of objects may also be required when a processor fails, when the current load on a processor is too high, or when the latency of a task is too high. The Resource Manager uses a Cooling Algorithm to migrate objects when the load on a processor is too high, and a Hot Spot Algorithm to migrate objects when the latency of a task is too high [8].

\subsubsection{Cooling Algorithm}

Periodically, the Resource Manager checks whether the load on a processor exceeds a predefined load limit, MAX_LOAD, determined by the application designer, or whether the load on the processor has increased considerably compared to the load on the other processors. If so, the Resource Manager determines whether any of the objects on that processor can be moved to the processor with the lowest load.

The Resource Manager identifies the processor low with the lowest load, the processor high with the highest load, 
and the processor high2 with the second highest load. It then calculates the contributions to the load on high made by the various objects on high. This load is derived from the mean processing time for the various methods of the object and the number of invocations per second of those methods by the various tasks. The objects are considered in the order of their loads. The Resource Manager then determines whether the candidate object $i$ can be moved to the least-loaded processor low without increasing the load on that processor above the allowable load. This continues until the load on processor high is below that of the second most highly loaded processor high 2 or until all of the objects have been considered.

\subsubsection{Hot Spot Algorithm}

Periodically, the Resource Manager estimates the projected latency for each task $t$, to check whether the latency of any task is too high a proportion of its deadline. If so, it searches for the object that is causing the largest queueing delay for task $t$ (the actual processing time cannot, of course, be reduced). This is the object $i$, executing on some processor $p$, whose methods $m$ cause, in aggregate, the largest increase in the latency to the completion of task $t$ because of queueing, computed as:

$$
\begin{aligned}
\text { Queueing_latency } y_{t i p} & \equiv \sum_{m \epsilon i} \frac{x_{t m} \tau_{m p}}{\left(1-\rho_{p}\right)}-x_{t m} \tau_{m p} \\
& =\sum_{m \in i} \rho_{p} \frac{x_{t m} \tau_{m p}}{\left(1-\rho_{p}\right)}
\end{aligned}
$$

where $m \in i$ indicates that $m$ is a method of object $i$.

The Resource Manager identifies the object $i$ that is causing the largest queueing delay for task $t$ and the processor $p$ on which object $i$ is executing. Once it has identified object $i$, it selects the processor low with the lowest load and attempts to move object $i$ to processor low. If this object migration would cause the load on processor low to exceed either the predefined load limit MAX_LOAD, or the allowable load on processor $p$, it attempts to move the next candidate object (causing the next largest queueing delay for task $t$ ). This continues until the Resource Manager determines an appropriate object to move. Once it has identified such an object, the Resource Manager moves that object from processor $p$ to processor low.

To estimate the effect of moving an object on the projected task latencies, the Resource Manager considers the effects of increased or decreased processor load on the times required to invoke the methods. It assumes an M/M/1 queueing model for each processor. The estimated time required for invocations of method $n$ on processor $p$ before moving object $i$ containing method $m$ to processor $p$ is:

$$
\frac{r_{n} \tau_{n p}}{1-\rho_{p}}
$$

After moving the object $i$ containing method $m$ to processor $p$, the estimated time is:

$$
\begin{aligned}
& \frac{r_{n} \tau_{n p}}{1-\rho_{p}-r_{m} \tau_{m p}} \\
& \quad=\frac{r_{n} \tau_{n p}}{1-\rho_{p}} * \frac{1-\rho_{p}}{1-\rho_{p}-r_{m} \tau_{m p}} \\
& \quad=\frac{r_{n} \tau_{n p}}{1-\rho_{p}} *\left(1+\frac{r_{m} \tau_{m p}}{1-\rho_{p}}+\left(\frac{r_{m} \tau_{m p}}{1-\rho_{p}}\right)^{2}+\ldots\right) \\
& \quad \approx \frac{r_{n} \tau_{n p}}{1-\rho_{p}} *\left(1+\frac{r_{m} \tau_{m p}}{1-\rho_{p}}\right)
\end{aligned}
$$

where the approximation is justified by the assumption that method $m$ contributes a small proportion of the total load on processor $p$, and is useful because it is expressed in terms of quantities measured by the Profilers. A similar estimate is made for the reduced time required for method invocations on the processor from which the object is moved.

Figure 4 shows how the projected latencies of Figure 3 are exploited for object allocation and reallocation. Objects are allocated or reallocated when a new application task is introduced into the system, when a processor has been lost due to a fault, when the load on some resource is too high, or when the latency for some task is too high. As the new application task runs, a profile of the method invocations for that task is constructed and is added to the Resource Manager's information base. New objects are tentatively allocated to to the most lightly loaded processors. This increases the load on the processor's resources and requires new projections of the task latencies. If the allocation is acceptable, it is actually performed. However, if a resource load is too high or a latency is too long, then objects are reallocated, or the degree of replication of an object is reduced, or the least important task is suspended. This process is repeated until an acceptable allocation is found, as shown by the solid lines in Figure 4.

\section{Replication in Realize}

In soft real-time distributed object systems, application objects can be replicated on different processors to provide fault tolerance and high availability. The degree of replication that is provided to an application object depends on the relative importance of that object to the application tasks. Application objects that have critical functionality are expected to be more highly replicated, while less important application objects might not be replicated at all.

In Realize, object replication, multicasting of invocations and responses, maintenance of replica consistency 


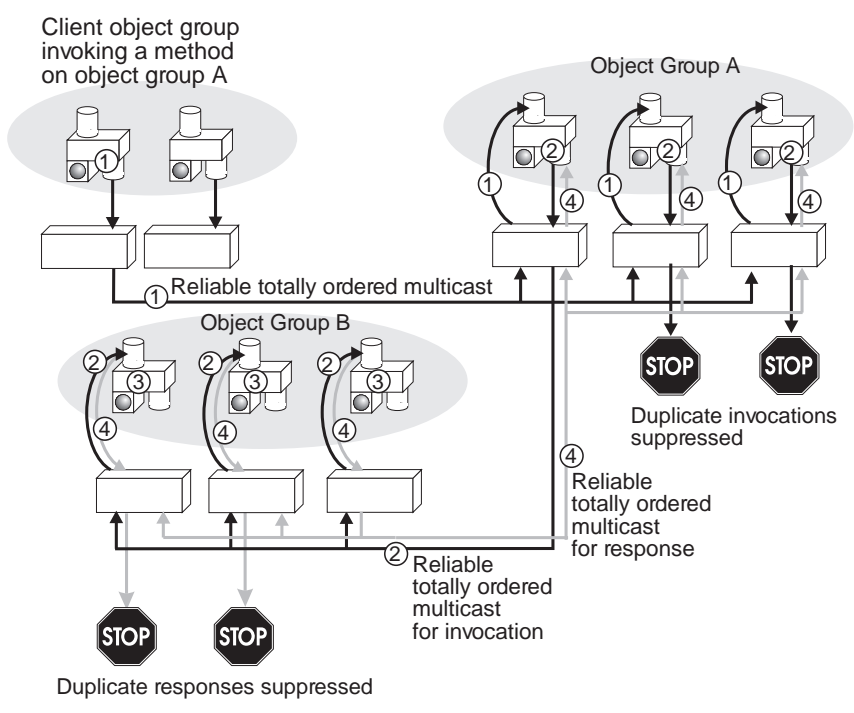

Figure 6: Active replication of objects within the Realize system.

and recovery from faults are transparent to the application objects and to the ORB. The type of replication and the reliability requirement, which implies the degree of replication, are determined for each object individually. Realize operates under the following assumptions:

- Independence of faults in the replicas of an object

- Deterministic behavior of the objects.

\subsection{Types of Replication}

The Replication Manager supports both active and passive replication, as shown in Figures 6 and 7. The application and the types of faults that must be tolerated dictate the type of replication that is employed.

\subsubsection{Active Replication}

In active replication, when a client object invokes a method on a server object, Realize multicasts the method invocation to the server object group via Totem and each server replica then executes the method, as shown in Figure 6. The underlying reliable totally ordered multicast protocol ensures that all of the replicas of an object receive the same messages in the same order and, thus, that they execute the methods in the same order. This ordering of method invocations ensures that the states of the replicas are consistent at the end of the operation.

\subsubsection{Passive Replication}

In passive replication, when a client object invokes a method on a server object, Realize multicasts the method invocation to that group via Totem and only one of the server replicas, the primary replica, executes the method, as shown in Figure 7. The Replication Manager at each of the other replicas logs the message containing the invocation so that one of those replicas can execute the method, should the primary replica fail. At the end of the method execution, Realize multicasts the updated state of the primary replica to the nonprimary replicas and multicasts the results to the client object via Totem. During the execution of the method, the states of the nonprimary replicas may differ from that of the primary replica; however, the state transfer achieves replica consistency at the end of the method execution.

\subsection{Fault Detection and Recovery}

Realize and Totem detect faults by using unreliable fault detectors based on timeouts. The failure of a processor or of the Replication Manager on a processor results in the failure of all object replicas on that processor.

For active replication, if any one of the replicas fails, service is uninterrupted (because the other replicas execute the method) and the results are returned to the invoking object. The replicas must also preserve the state of the object for subsequent transfer to a new or recovered replica.

For passive replication, the effect of a fault depends on whether the failed replica is a primary or nonprimary replica. The failure of a nonprimary replica during the execution of a method is transparent to a client, because Realize removes that replica from the group, while the primary replica continues to execute the method.

If the primary replica fails, Realize detects the fault and determines a new primary replica. The new primary replica must execute the method invocations that it has logged and for which it has not received a response and state transfer from the prior primary replica. Invocations on other objects, and even responses, can be generated by both the original and the new primary replicas. The Replication Manager suppresses such duplicate invocations and duplicate responses.

\subsection{Replica Consistency}

To ensure that the replicas of an object are updated consistently, Realize exploits the reliable totally ordered message delivery service of an underlying group communication system, in our case Totem [12]. The invocations of methods on the replicas, and the corresponding responses, are contained in multicast messages. Because the messages are delivered in the same total order at each object replica, the methods are executed by each replica in the same order, thereby maintaining consistency of the states of the replicas.

For active replication, this ensures that the invocation of a method, and the corresponding response, is received by all of the replicas of an object or by none of them. Consequently, the method is executed by all of the replicas or by none of them. For passive replication, this ensures that either all of the nonprimary replicas have the updated 


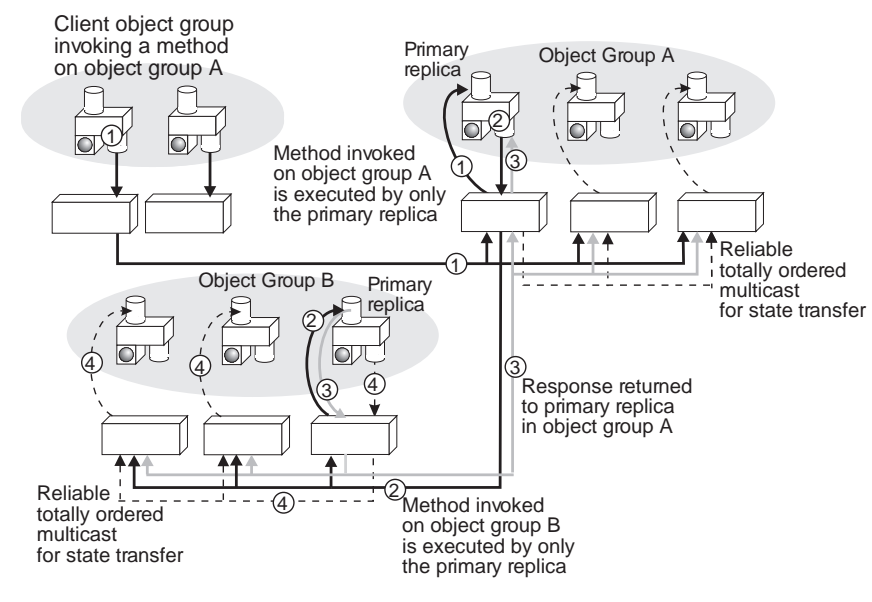

Figure 7: Passive replication of objects within the Realize system.

state of the object or, alternatively, all of the nonprimary replicas know that none of them has the updated state.

A reliable totally ordered message delivery service alone does not suffice to maintain the consistency of the states of the replicas. To maintain replica consistency, Realize also detects and suppresses duplicate operations and transfers state between replicas, as shown in Figure 6. It also ensures that, at any time, only one invocation is being processed within each process, reordering messages as necessary to ensure this. More details of the replica consistency mechanisms used by Realize can be found in $[11,13,15]$.

\subsection{Degree of Replication}

Realize maintains the degree of replication of the application objects to achieve the desired level of reliability, while allocating sufficient resources to the objects to guarantee a certain quality of service. When computing resources are limited, increasing the degree of replication of one application object may adversely affect the quality of service provided to another application object.

Each application object requires a certain minimum amount of resources to perform acceptably. When spare resources are available, those resources can be used for additional object replicas. In such cases, even the small value of one additional replica is greater than the value of an idle resource. On the other hand, when resources are scarce and a large number of application objects are competing for resources, the decision as to which application objects to replicate, if any, is not obvious.

Realize aims to determine the degree of replication of each application object, and to allocate resources to each application task, so as to maximize the system utility while respecting the resource constraints.

As new application tasks are introduced into the system, the Resource Manager determines, using the Profilers' information, whether it can satisfy the requests, determines the replication degree for the application objects, and allocates resources accordingly.

During operation, if insufficient resources remain to run the application tasks or to provide the quality of service they require, the Resource Manager attempts to reallocate the object replicas dynamically to free computing resources by migrating them to other processors in the system; otherwise, it reduces their degree of replication. If a processor or other resource is lost due to a fault, the Resource Manager recomputes the number of replicas for each application object, which may involve reducing the degree of replication of certain objects or reallocating object replicas to adjust to new resource constraints. When a new object is introduced into the system, it may be necessary to remove an existing object replica to allocate resources to the new object.

\subsubsection{The Model}

The distributed system includes two types of resources: memory and CPUs. Each resource can be used by multiple tasks, and each task individually contributes only a small proportion of the total load on the resources. We let $M_{p}$ denote the total memory on processor $p, L_{p}$ the maximum CPU capability of processor $p, h_{p}$ the proportion of the memory that is allowed to be used, and $h_{p}^{\prime}$ the proportion of the CPU that is allowed to be used. For instance, if the utilization of all CPUs should be kept lower than $70 \%$, then $h_{p}^{\prime}$ is set to 0.7 for each processor $p$.

Each application task is a sequence of method invocations of possibly different objects between the acceptance of an external input (or possibly a timer signal or completion of another operation) and the generation of an external result or signal. The tasks arrive independently but their sets of method invocations are not necessarily disjoint. Thus, although each application object is created by one task, its methods may be invoked by several different tasks.

In the ideal case, the system can accommodate at least one replica for the objects of all of the application tasks that require service. The limited amount of system resources requires determination of whether the object of an application task should be replicated, based on the relative importance of the task for the system. The Importance $t_{t}$ of task $t$ is defined as a reward $g_{t}$ gained from the execution of task $t$. No penalty is associated with the absence of the execution of a task. An application task completes successfully if each method invoked by the task completes execution of at least one replica of each of the objects successfully. All of the replicas of an object are independent and provide the same functionality.

Assuming that the individual replicas of an object fail independently with equal probabilities, the probability that all replicas of an object fail diminishes as the number of replicas increases. We let $q_{i}$ denote the probability that a 
single replica of object $i$ fails and $r_{i}$ the number of replicas of object $i$. The Reward $G_{i}$ of object $i$ is the sum of the rewards $g_{t}$ of all application tasks $t$ that invoke object $i$ :

$$
G_{i}=\sum_{i: i \in t} g_{t}
$$

where $i \in t$ denotes that task $t$ invokes a method of object $i$.

The Utility $U_{i_{r}}$ of the $i_{r}$ th replica of object $i$ is defined to be the product of object $i$ 's reward and the probability of failure of all but one of the replicas:

$$
U_{i_{r}}=G_{i} *\left(\begin{array}{c}
i_{r} \\
i_{r}-1
\end{array}\right) * q_{i}^{i_{r}-1} *\left(1-q_{i}\right)
$$

Thus, the utility of the first replica of object $i$ is $G_{i}$, the utility of the second replica is the product of $G_{i}$ and the probability that only one of the two replicas has failed, the utility of the third replica is the product of $G_{i}$ and the probability that any two of the three replicas have failed, etc. These utilities can be computed quite quickly and stored in a table.

The System_utility $U(\bar{r})$ is the sum of the utilities of the object replicas:

$U(\bar{r})=\sum_{i} U_{i_{r}}=\sum_{i} \sum_{i_{r}} G_{i} *\left(\begin{array}{c}i_{r} \\ i_{r}-1\end{array}\right) * q_{i}^{i_{r}-1} *\left(1-q_{i}\right)$

where $\bar{r}=\left(r_{1}, r_{2}, \ldots, r_{i}, \ldots\right)$ and $r_{i}$ is the number of replicas of object $i$.

We let $m_{p i}$ denote the proportion of memory used by object $i$ on processor $p$ and $\ell_{p t i}$ the load that object $i$ invoked by task $t$ imposes on processor $p$. The load that object $i$ imposes on $p$ is derived from the Mean_processing_time $\tau_{\text {wip }}$ required for a method $w$ of object $i$ executing on processor $p$, the mean number $x_{t i w}$ of invocations of method $w$ of object $i$ by task $t$, and the Projected_latency $T_{t}$ of task $t$. Thus, the Load $\ell_{p t i}$ of an object $i$ on processor $p$ invoked by task $t$ is computed as:

$$
\ell_{p t i}=\frac{\sum_{w} \tau_{w i p} * x_{t i w}}{T_{t}}
$$

Assuming that it is always possible to recreat at least one replica of each object, the following resource utilization constraints must be satisfied. For each processor $p$,

$$
\begin{aligned}
\sum_{i: i \in p} m_{p i} & \leq h_{p} M_{p} \\
\sum_{t} \sum_{i: i \in t \wedge i \in p} \ell_{p t i} & \leq h_{p}^{\prime} L_{p}
\end{aligned}
$$

The quantities in these inequalities are obtained either from measurements and computations $\left(G_{i}, m_{p i}, \ell_{p t i}, q_{i}\right)$ performed by the Profilers and the Resource Manager or from information $\left(M_{p}, L_{p}\right)$ specified by the application designer.

We consider the problem of choosing the combination of numbers of replicas that maximizes the System_utility $U(\bar{r})$, subject to the above resource utilization constraints. For each object, at least one replica is chosen. Then, depending on the availability of resources in the system, additional replicas are chosen with the aim of maximizing the system utility $U(\bar{r})$.

\subsubsection{Greedy Algorithm}

The above maximization problem is NP hard. An exact solution requires an exhaustive search that considers all possible combinations, which is too expensive for a dynamic allocation algorithm. Furthermore, the values of the importance of the objects and the rewards of the tasks are only estimates. Consequently, the cost of finding an exact solution is unjustified.

Therefore, we employ a greedy algorithm, where the Load $\ell_{p i}$ of an object $i$ on processor $p$ for all tasks $t$ executing on processor $p$ is given by:

$$
\ell_{p i}=\sum_{t: i \in t, i \in p} \ell_{p t i}
$$

and the available load AvailLoad $d_{p}$ on processor $p$ is given by:

$$
\operatorname{AvailLoad}_{p}=h_{p}^{\prime} L_{p}-\sum_{t} \sum_{i: i \in t \wedge i \in p} \ell_{p t i}
$$

and the available memory $A$ vail $\mathrm{Mem}_{p}$ on processor $p$ is given by:

$$
\operatorname{AvailMem}_{p}=h_{p} M_{p}-\sum_{i: i \in p} m_{p i}
$$

The greedy algorithm chooses incrementally the unallocated replica with the highest utility and allocates it to the least loaded processor, subject to the resource constraints. In effect, at each step the greedy algorithm determines a local maximum for the system utility function. The final resulting value of the system utility function may not be a global maximum.

The Resource Manager invokes the greedy algorithm when objects are replicated initially and when the system conditions change considerably. Replicas of existing objects may be removed when a new task starts or when resource capacities have been exceeded.

\section{Related Work}

For a real-time distributed system with two or more processors, it has been shown that no deadline scheduling algorithm can be optimal without complete a priori knowledge of the arrival times, computation times, and deadlines 
of future tasks [4]. They have shown that least laxity scheduling is an effective strategy for real-time distributed systems, provided that the system is not overloaded. Realize uses least laxity scheduling.

Several efforts are underway to extend CORBA with real-time and fault-tolerance capabilities, including work of the Object Management Group (OMG) Realtime Platform Special Interest Group [17, 18, 20, 21], in which we are participating. As yet, the OMG specifications provide only static fixed priority scheduling.

Gill, Levine, Schmidt and Kuhns [6] have developed a maximum urgency first scheduling algorithm for the TAO real-time ORB [24] that provides scheduling assurances for critical tasks while optimizing the use of scarce resources. Sydir, Chatterjee and Sabata [27] have implemented an endto-end QoS-driven resource management scheme within the ERDoS ORB, that considers the QoS requirements and resource requirements of each object individually. Wolfe, et al [29] have introduced timed distributed method invocations as a real-time CORBA extension to enable the expression and enforcement of timing constraints in client/server interactions by attaching a separate object with timing characteristics to each method invocation.

Nett, Gergeleit and Mock [16] have developed an adaptive system using integrated monitoring, dynamic execution time prediction and scheduling to provide time-awareness for standard CORBA method invocations. Rosu, Schwan and Jha [23] have introduced adaptive resource allocation mechanisms that can adjust resource allocation to changes in the applications' needs and have proposed a set of performance metrics for capturing the impact of those mechanisms on the performance of the applications. Rajkumar, Lee, Lehoczky and Siewiorek [22] have developed the Q-RAM model that focuses on allocating multiple resources to multiple applications such that each application can meet its minimum needs and yet maximize the system utility.

Other researchers have developed middleware that extends CORBA with fault tolerance. The Electra toolkit $[9,10]$ built by Maffeis on top of Horus provides support for replicated CORBA objects, as does Orbix+Isis [9] on top of Isis. Unlike Realize, Electra and Orbix+Isis are nonhierarchical object systems and support only active replication. Both Electra and Orbix+Isis use an integration approach in that the replication and group communication mechanisms are integrated into the ORB and require modification of the ORB. In contrast, Realize uses the interception approach, which requires no modification to the ORB.

The Distributed Object-Oriented Reliable Service (DOORS) [25] augments CORBA with fault tolerance using passive replication by providing replica management, fault detection, and fault recovery as CORBA service objects above the ORB. The DoorMan management interface monitors DOORS and the underlying system to take corrective action by migrating objects, if their hosts are suspected of being faulty. The OpenDREAMS toolkit [5] also uses the service approach but provides support for both active and passive replication by adding replication and group communication as service objects on top of the ORB. The service approach requires no modification of the ORB, but lacks the transparency and efficiency that Realize provides.

The AQuA framework [3] employs the Quality of Service for CORBA Objects $(\mathrm{QuO})$ runtime, the Proteus dependability property manager, and the Maestro/Ensemble toolkits. QuO [30] extends the Interface Definition Language (IDL) of CORBA with a Quality of Service Description Language (QDL). It provides mechanisms for measuring and enforcing QoS contracts and taking appropriate actions when those contracts are violated. Based on the requirements communicated by the $\mathrm{QuO}$ runtime and the faults that occur, the Proteus dependability property manager determines the type of faults to tolerate, the replication policy, the degree of replication, and the location of the replicas, and dynamically modifies the configuration to meet those requirements. The Maestro/Ensemble toolkits [28] uses a gateway to translate CORBA object invocations into messages that are transmitted via the Ensemble group communication system, and detects and filters duplicate invocations (responses). AQuA is substantially less transparent to the application than is Realize.

Shokri, Hecht, Crane, Dussault and Kim [26] have observed that effective fault handling in complex distributed applications requires the ability to adapt resource allocation and fault-tolerance policies dynamically in response to changes in the environment, application requirements and available resources. Bondavalli, Giandomenico and Mura [2] have defined a planner component based on a valuedriven admission policy that minimizes the effects that high reliability requirements have on the expected quality of service and achieves the optimal utilization of available system resources.

\section{Conclusion}

The Realize system simplifies the development of complex distributed defense systems by extending the services of CORBA with resource management, soft real-time scheduling and fault tolerance.

The Resource Manager employs a three-level feedback loop structure, based on the profiling of the application objects, which allocates objects to processors and migrates them between processors to balance the load across the system. It also provides least-laxity scheduling to meet soft real-time deadlines for tasks that span multiple processors.

The Replication Manager replicates objects, using either active or passive replication as appropriate, and maintains the consistency of the states of the replicas using reliable 
totally ordered multicast messages. Realize determines the appropriate degree of replication of objects, balancing fault-tolerance and real-time performance requirements.

Thus, Realize simplifies the programming of soft realtime fault-tolerant distributed applications by hiding the difficult issues of distribution, real-time scheduling, load balancing, replication, consistency, fault detection and recovery, allowing the application programmer to focus on the application. Realize maintains the portability, location transparency and interoperability that COTS implementations of CORBA provide, and has been demonstrated with seven unmodified commercial CORBA ORBs, incurring an additional overhead of only about $10-15 \%$.

\section{References}

[1] R. Bettati and A. Gupta, "Dynamic resource migration for multiparty real-time communication,' Proceedings of the IEEE 16th International Conference on Distributed Computing Systems (May 1996), Hong Kong, pp. 646-655.

[2] A. Bondavalli, F. D. Giandomenico and I. Mura, "Value-driven assignment in object-oriented realtime dependable systems," Proceedings of the IEEE 3rd International Workshop on Object-Oriented RealTime Dependable Systems, Newport Beach, CA (February 1997), pp. 92-99.

[3] M. Cukier, J. Ren, C. Sabnis, W. H. Sanders, D. E. Bakken, M. E. Berman, D. A. Karr and R. E. Schantz, "AQuA: An adaptive architecture that provides dependable distributed objects,' Proceedings of the IEEE 17th Symposium on Reliable Distributed Systems, West Lafayette, IN (October 1998), pp. 245253.

[4] M. L. Dertouzos and A. K. Mok, "Multiprocessor on-line scheduling of hard-real-time tasks,' IEEE Transactions on Software Engineering, vol. 15, no. 12 (December 1989), pp. 1497-1506.

[5] P. Felber, B. Garbinato and R. Guerraoui, 'Designing a CORBA group communication service,', Proceedings of the IEEE 15th Symposium on Reliable Distributed Systems, Niagara on the Lake, Canada (October 1996), pp. 150-159.

[6] C. Gill, D. L. Levine, D. C. Schmidt and F. Kuhns, "Evaluating strategies for real-time CORBA dynamic scheduling,' International Journal of Time-Critical Computing Systems, to appear.

[7] V. Kalogeraki, L. E. Moser and P. M. Melliar-Smith, "Dynamic modeling of replicated objects for dependable soft real-time distributed object systems,', Proceedings of the IEEE 4th Workshop on Object-
Oriented Real-time Dependable Systems, Santa Barbara, CA (January 1999), pp. 48-55.

[8] V. Kalogeraki, P. M. Melliar- Smith and L. E. Moser, "Using multiple feedback loops for object profiling, scheduling and migration in soft real-time distributed object systems," Proceedings of the IEEE 2nd International Symposium on Object-Oriented Real-Time Distributed Computing, Saint Malo, France (May 1999), pp. 291-300.

[9] S. Landis and S. Maffeis, "Building reliable distributed systems with CORBA,' Theory and Practice of Object Systems, vol. 3, no. 1, pp. 31-43.

[10] S. Maffeis, '“Electra --- Making distributed programs object-oriented,' Proceedings of the USENIX Symposium on Experiences with Distributed and Multiprocessor Systems, vol. IV, San Diego, CA, pp. 143-156.

[11] P. M. Melliar-Smith, L. E. Moser, V. Kalogeraki and P. Narasimhan "The Realize middleware for replication and resource management,' Proceedings of the IFIP International Conference on Distributed Systems Platforms and Open Distributed Processing, Middleware '98, The Lake District, England (September 1998), pp. 123-138.

[12] L. E. Moser, P. M. Melliar-Smith, D. A. Agarwal, R. K. Budhia and C. A. Lingley-Papadopoulos, "Totem: A fault-tolerant multicast group communication system,' Communications of the ACM, vol. 39, no. 4 (April 1996), pp. 54-63.

[13] L. E. Moser, P. M. Melliar-Smith and P. Narasimhan, " Consistent object replication in the Eternal system,', Theory and Practice of Object Systems, vol. 4, no. 2, pp. 81-92.

[14] P. Narasimhan, L. E. Moser and P. M. Melliar-Smith, "Exploiting the Internet Inter-ORB protocol interface to provide CORBA with fault tolerance,' 'Proceedings of the USENIX 3rd Conference on ObjectOriented Technologies and Systems, Portland, OR (June 1997), pp. 81-90.

[15] P. Narasimhan, L. E. Moser P. M. and Melliar-Smith, "Replica consistency of CORBA objects in partitionable distributed systems,' Distributed Systems Engineering, vol. 4, no. 3 (September 1997), pp. $139-150$.

[16] E. Nett, M. Gergeleit and M. Mock, “An adaptive approach to object-oriented real-time computing,' Proceedings of the IEEE 1st International Symposium on Object-Oriented Real-Time Distributed Computing, Kyoto, Japan (April 1998), pp. 342-349. 
[17] Object Management Group, "Fault Tolerance for CORBA," OMG Technical Committee Document orbos/98-10-08 (October 1998).

[18] Object Management Group, "Realtime CORBA," OMG Technical Committee Document orbos/99-0212 (February 1999).

[19] Object Management Group, "The Common Object Request Broker: Architecture and Specification," 2.3 edition, OMG Technical Committee Document formal/98-12-01 (June 1999).

[20] Object Management Group, "Fault Tolerant CORBA," OMG Technical Committee Document orbos/99-10-05 (October 1999).

[21] Object Management Group, "Dynamic Scheduling," OMG Technical Committee Document orbos/99-1006 (October 1999).

[22] R. Rajkumar, C. Lee, J. Lehoczky and D. Siewiorek, "A resource allocation model for QoS management," Proceedings of the IEEE 18th Real-Time Systems Symposium, San Francisco, CA (December 1997), pp. 298-307.

[23] D. I. Rosu, K. Schwan and R. Jha, "On adaptive resource allocation for complex real-time applications," Proceedings of the IEEE 18th Real-Time Systems Symposium, San Francisco, CA (December 1997), pp. 320-329.

[24] D. C. Schmidt, D. L. Levine and S. Mungee, "The design of the TAO real-time object request broker," Computer Communications, vol. 21, no. 4 (April 1998) pp. 294-324.
[25] J. Schonwalder, S. Garg, Y. Huang, A. P. A. van Moorsel and S. Yajnik, "A management interface for distributed fault tolerance CORBA services," Proceedings of the IEEE 3rd International Workshop on Systems Management, Newport, RI (April 1998), pp. 98-107.

[26] E. Shokri, H. Hecht, P. Crane, J. Dussault and K. H. Kim, "An approach for adaptive fault-tolerance in object-oriented open distributed systems," Proceedings of the IEEE 3rd International Workshop on Object-Oriented Real-Time Dependable Systems, Newport Beach, CA (February 1997), pp. 298-305.

[27] J. J. Sydir, S. Chatterjee and B. Sabata, "Providing end-to-end QoS assurances in a CORBA-based system," Proceedings of the IEEE 1st International Symposium on Object-Oriented Real-Time Distributed Computing, Annapolis, MA (May 1998), pp. 10-17.

[28] A. Vaysburd and K. Birman, "The Maestro approach to building reliable interoperable distributed applications with multiple execution styles," Theory and Practice of Object Systems, vol. 4, no. 2 (1998), pp. 73-80.

[29] V. F. Wolfe, L. C. DiPippo, R. Ginis, M. Squadrito, S. Wohlever, I. Zykh, and R. Johnston, "Expressing and enforcing timing constraints in a dynamic real-time CORBA system,"' Technical Report, Department of Computer Science and Statistics, University of Rhode Island (1997).

[30] J. Zinky, D. Bakken and R. Schantz, "Architectural support for quality of service for CORBA objects" Theory and Practice of Object Systems, vol. 3, no. 1 (1997), pp. 55-73. 